OITS-676

RU-99-25

\title{
Magnetic Interactions, the Renormalization Group and Color Superconductivity in High Density QCD
}

\author{
Stephen D.H. Hsu* \\ Department of Physics, University of Oregon, Eugene OR 97403-5203. \\ Myckola Schwetz ${ }^{\dagger}$ \\ Department of Physics and Astronomy, Rutgers University, Piscataway NJ 08855-0849.
}

August 1999

\begin{abstract}
We investigate the effect of long range magnetic interactions on the renormalization group (RG) evolution of local Cooper pairing interactions near the Fermi surface in high density QCD. We use an explicit cut-off on momentum modes, with special emphasis on screening effects such as Landau damping, to derive the RG equations in a gauge invariant, weak coupling expansion. We obtain the Landau pole $\Delta \sim \mu g^{-5} \exp \left(-\frac{3 \pi^{2}}{\sqrt{2} g}\right)$, although the structure of our equations differs from previous results. We also investigate the gap equation, including condensates of higher angular momentum. We show that rotational invariance is unbroken at asymptotically high density, and verify that $\Delta$ is the correct value of the gap when higher modes are included in the analysis.
\end{abstract}

*hsu@duende.uoregon.edu

†myckola@baobab.rutgers.edu 


\section{Introduction}

In this paper we study the behavior of quark matter at high density and low temperature [1]-18. Under these conditions QCD exhibits color superconductivity, caused by the condensation of diquarks. This condensation is analogous to the Cooper pairing observed in ordinary superfluids, and can be shown to occur in the presence of even very weakly attractive interactions. The reason for this is the special nature of physics very close to the Fermi surface (FS).

At high density, characterized by a chemical potential $\mu$ which is much larger than the cur-

rent quark masses and the QCD scale $\Lambda_{Q C D}$, the typical momentum transfer in quark-quark interactions is of order $\mu$, and therefore it is plausible that the dynamics can be understood via perturbative gluon exchange. Of course, small angle scatterings, which involve small momentum transfers, are still problematic and require special attention.

In recent work, Son [7] showed that long range magnetic interactions lead to a modification of the RG equations originally derived in [5] for the case of local interactions. The magnetic effects are strong enough to modify the parametric dependence of the position of the Landau pole (and hence the superfluid gap $\Delta$ ) on the gauge coupling constant. Son finds

$$
\Delta \sim \mu g^{-5} \exp \left(-\frac{3 \pi^{2}}{\sqrt{2} g}\right) \quad
$$

That $\Delta$ should scale like $\exp (-c / g)$ is easy to see [\&] by considering the gap equation with a massless, or weakly damped, gauge propagator. In the usual case of a local four fermion interaction, the gap integral exhibits a logarithmic divergence which, roughly speaking, is cut off near the FS by the gap itself. Solving for $\Delta$ yields a result of the form $\Delta \sim \exp (-c / G)$, where $\mathrm{G}$ is the four fermion coupling, and is of order $g^{2}$ if it arises from the exchange of a gauge boson. However, if the four fermion interaction is replaced by a weakly damped gauge propagator, an additional logarithmic divergence appears due to small angle scattering of the fermions. This divergence is again regulated by a scale related to the gap itself, and the resulting exponent of the solution is roughly the square root of the what appeared in the local case: $\Delta \sim \exp (-c / g)$.

Our intention here is to understand this behavior in terms of the evolution of Cooper pairing interactions near the Fermi surface. Despite the long range of the magnetic interactions, we find that the problem can still be formulated in terms of local operators which are, essentially, terms in the expansion of the magnetic gluon form factor. The reason that this is possible is because we retain at all times an explicit cutoff on long wavelength modes, which keeps all quantities finite. This approach is somewhat different from that of Son [7], who studied the RG evolution of scattering amplitudes themselves. As discussed below, 
our results differ from his. Primarily, we believe that this is due to conceptual problems in applying the RG directly to scattering amplitudes.

The paper is organized as follows. In section 2 we give a description of our cutoff scheme and the resulting effective lagrangian. In section 3 we discuss the problem of small angle scattering and gluon screening effects. In section 4 we compute our RG equations and compare them with Son's. In section 5 we investigate the possibility of breaking rotational symmetry. The final section contains additional discussion of our results.

\section{RG Scheme}

We adopt a Wilsonian RG procedure, with a hard IR cutoff on spatial momentum, $\Lambda$ [5, 19]. As $\Lambda \rightarrow 0$, only quark excitations very near the FS, as well as soft gluons, are left in the effective theory. Our prescription differs from what is often used in QFT, where a hard cutoff is imposed on energy as well as momentum, but it has some advantages. In particular, in our scheme integration over modes corresponds to shrinking the Hilbert space of the model in the basis of energy eigenstates.

The effective Lagrangian has the form

$$
\mathcal{L}_{\Lambda}=\mathcal{L}_{Q C D}+\sum_{n} \mathcal{O}_{n}
$$

where the $\mathcal{O}_{n}$ are local operators involving quark and gluon fields, which are the result of the integration over higher frequency quarks and gluons. The most important of these operators are the marginal Cooper pairing interactions which involve four quarks. All other quark interactions can be shown to be irrelevant in the limit of small $\Lambda$ [5].

The Cooper pairing interaction is of the form

$$
G(k-q) \bar{\psi}_{+}\left(k_{0}, k\right) \gamma_{\mu} P_{L, R} \psi_{+}\left(q_{0}, q\right) \bar{\psi}_{+}\left(k_{0},-k\right) \gamma^{\mu} P_{L, R} \psi_{+}\left(q_{0},-q\right)
$$

where $\psi_{+}$denotes the projection of the quark field

$$
\psi_{+}=\frac{1}{2}(1+\vec{\alpha} \cdot \hat{p}) \psi(p)
$$

and consists of quark, rather than antiquark degrees of freedom. We will be interested in the case where all of the external quarks in this operator are essentially on-shell. Note that the incoming and outgoing quarks have almost equal and opposite momenta. Near the FS, the form factor $G(k-q)$ becomes a function of angle $\theta=\frac{\vec{k} \cdot \vec{q}}{k q}$, since $|k| \simeq|q| \simeq \mu$, and $k_{0}, q_{0} \simeq 0$. (Strictly speaking, in the case of Landau damping, it is a function of energy as well as angle. 
We will always assume that the energy transfer $k_{0}-q_{0}$ in the gluon line is much less than but of order $\Lambda$.) In this paper we restrict ourselves to the $\overline{3}$ color channel, which is attractive and to the LL (or equivalently RR) chirality channel, which has been shown to dominate the LR channel [5]. It is straightforward to derive the related RG equations for other channels using our techniques.

In previous work we took the form factor $G(\theta)$ to be a constant [5]. This is appropriate at sufficiently low energy, if screening masses exist for both the magnetic and electric components of the gluon, which is likely to be the case at intermediate densities where the coupling is not small. However, as argued by Son [7], a magnetic mass for the gluon is unlikely to arise within perturbation theory. The magnetic mass is due solely to nonperturbative effects, and is presumably of order $\exp \left(-1 / g^{2}\right)$. At high density it is therefore likely to be smaller than the eventual superfluid gap, and hence plays no role in the analysis. Instead, [7] focused on the role of Landau damping on the magnetic interactions.

In order to consider long range magnetic interactions, it is necessary to expand the form factor $G(\theta)$ in components with definite angular momentum. We can then study the RG evolution of each of these components. Let

$$
G(\theta)=\sum_{l}(2 l+1) P_{l}(\cos \theta) G_{l}
$$

so that each component

$$
G_{l}=\frac{1}{2} \int_{-1}^{1} d(\cos \theta) P_{l}(\cos \theta) G(\theta)
$$

This integral exhibits a logarithmic divergence in the case of a massless, or weakly damped, gluon (figure [1). However, in our regularization scheme $G(\theta)$ contains only the effects of gluons which have been integrated out above the cutoff $\Lambda$. For nonzero cutoff the components $G_{l}$ will be finite, but exhibit a logarithmic dependence on $\Lambda$ ?. It is this logarithmic dependence that leads to the constant $\mathcal{O}\left(g^{2}\right)$ term in the RG equations noted by Son [7].

\section{$3 \quad$ Screening Effects}

In this section we discuss the incorporation of screening effects in our procedure. As mentioned previously, small angle scattering of quarks must be considered carefully, as the simple perturbative expansion in powers of $g^{2}$ may break down. Indeed, it is easy to see that vacuum polarization corrections to any graph can become important if the gluon momentum is

\footnotetext{
${ }^{1}$ The precise form of this logarithm is dependent on the IR behavior of the propagator, as we will see below.
} 


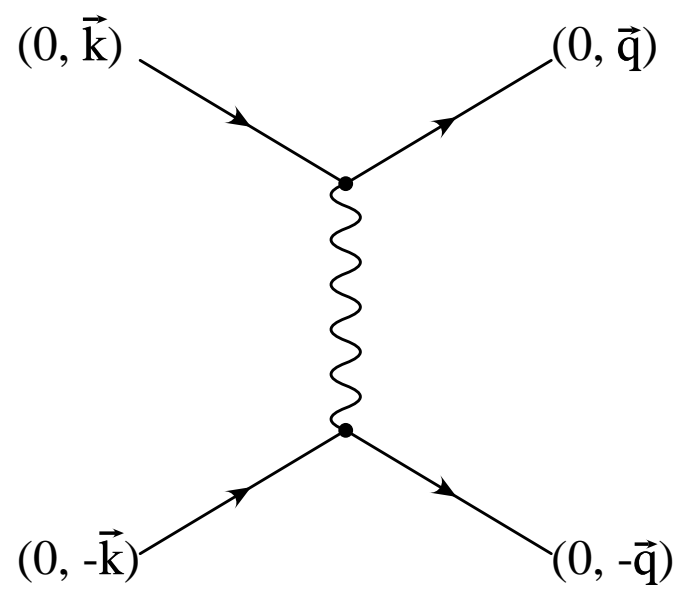

Figure 1: One gluon exchange.

sufficiently small. Resummation of these effects leads to a screened propagator which is well behaved at small momentum, as long as the energy is nonzero.

The gluon propagator, including vacuum polarization effects from virtual quarks, 20] has the following form in covariant gauge:

$$
D_{\mu \nu}=\frac{1}{G+Q^{2}} P_{\mu \nu}^{T}+\frac{1}{F+Q^{2}} P_{\mu \nu}^{L}-\xi \frac{Q_{\mu} Q_{\nu}}{Q^{4}},
$$

where $Q=\left(q_{4}, \vec{q}\right)=(-\omega, \vec{q})$ is the gluon Euclidean 4-momentum, and $P_{\mu \nu}^{T}$ and $P_{\mu \nu}^{L}$ are transverse and longitudinal projectors correspondingly. In our leading order calculations the propagator will always appear contracted with gamma matrices next to on-shell external quark lines. Thus the gauge dependent part of (7) will vanish due to the equations of motion, leading to a gauge-invariant result. Henceforth we will simply set $\xi=0$ in (7).

The functions $G$ and $F$ are related to the gluon polarization tensor $\Pi_{\mu \nu}$ :

$$
\begin{aligned}
F & =\frac{Q^{2}}{q^{2}} \Pi_{44}, \\
G & =\frac{1}{2} P_{\mu \nu}^{T} \Pi_{\mu \nu}=\frac{1}{2}\left(\delta_{i j}-\frac{q_{i} q_{j}}{q^{2}}\right) \Pi_{i j},
\end{aligned}
$$

which reflects the fact that the Lorentz symmetry is broken to 3D rotational symmetry. The explicit expression of $\Pi_{\mu \nu}$ to one loop is

$$
\Pi_{\mu \nu}(Q)=g^{2} \int \frac{d^{4} K}{(2 \pi)^{4}} \operatorname{Tr}\left[\gamma_{\mu} \not K \gamma_{\nu}(\not K-\not Q)\right] \Delta(K) \Delta(K-Q),
$$

where $\Delta(K)=1 / K^{2}$. 


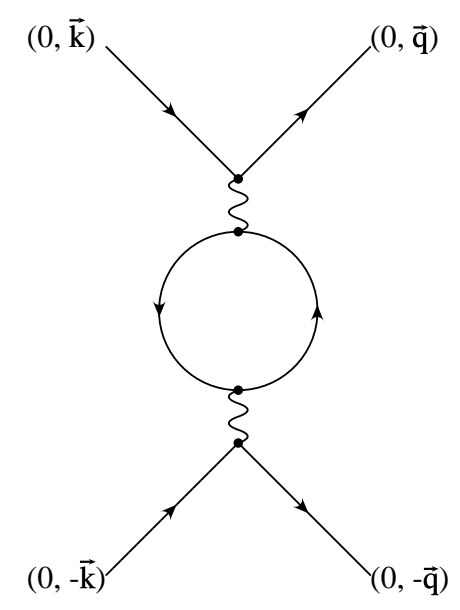

Figure 2: Vacuum polarization correction to gluon propagator.

If the energy and momentum transfer in figure (22) are small then one may neglect $Q$ in the numerator of (8), as the dominant momenta in the loop will be $k \sim \mu$ (this is the equivalent of the hard thermal loop approximation). Then $F$ and $G$ take the familiar form 20

$$
\begin{aligned}
& F=\frac{2 m_{D}^{2} Q^{2}}{q^{2}}\left(1-\frac{i \omega}{q} L_{0}\left(\frac{i \omega}{q}\right)\right) \\
& G=m_{D}^{2} \frac{i \omega}{q}\left[\left(1-\left(\frac{i \omega}{q}\right)^{2}\right) L_{0}\left(\frac{i \omega}{q}\right)+\frac{i \omega}{q}\right],
\end{aligned}
$$

where

$$
L_{0}(x)=\frac{1}{2} \ln \frac{x+1}{x-1},
$$

and $m_{D}^{2}=N_{f} \frac{g^{2} \mu^{2}}{2 \pi^{2}}$ is the Debye screening mass. The small x expansion of $\mathrm{G}$ leads to the Landau damped magnetic gluon propagator

$$
D_{\mu \nu}^{T}\left(q_{0}, q\right)=\frac{P_{\mu \nu}^{T}}{q^{2}+i \frac{\pi}{2} m_{D}^{2} \frac{\left|q_{0}\right|}{q}}
$$

while the expansion of $\mathrm{F}$ leads to the usual longitudinal propagator, with Debye screening. The effect of Landau damping is to cut off the small-q divergence in (11) at $q \sim q_{0}^{1 / 3} m_{D}^{2 / 3}$.

One must be careful to compute the screening effects using a Wilsonian cutoff. When we integrate over a shell in momentum space only the contribution of quarks which have already been integrated out is to be included in the screening effects. This means that we must re-examine the calculation which leads to the Debye mass and Landau damping, and use cutoff-dependent versions of the vacuum polarization $\equiv \Pi_{\mu \nu}^{\Lambda}$ in our RG. 
The terms in $\mathrm{F}$ and $\mathrm{G}$ can be shown to result from integration of quark modes within roughly $q$ of the FS. For example, the Landau damping term originates from an integral of the form (arising from (8) 20]):

$$
I(\omega, \vec{q})=\int d k d \Omega \frac{k^{4}}{E_{1} E_{2}}\left[\frac{n\left(E_{1}\right)-n\left(E_{2}\right)}{i \omega-E_{1}+E_{2}}\right],
$$

where $E_{1}=k, E_{2}=|\vec{k}-\vec{q}| \simeq k-q \cos \theta$ and

$$
n(E)=\frac{1}{e^{\beta(E-\mu)}+1}
$$

is the Fermi-Dirac distribution for quarks. $n(E)$ is a theta function of $(\mu-E)$ in the low temperature limit in which we work. For a given value of $\theta=\frac{\vec{k} \cdot \vec{q}}{k q}$, the modes which contribute to Landau damping must be within $\simeq q \cos \theta$ of the FS due to the theta functions.

If the momentum transfer $q<\Lambda<<\mu$, then none of these modes are included in the calculation of $\Pi_{\mu \nu}^{\Lambda}$. The corresponding $F^{\Lambda}$ and $G^{\Lambda}$ are changed drastically: in particular the leading $\mu^{2}$ behavior of $F$ vanishes, and $G$ becomes proportional to $\mu^{2}$. Fortunately, our interest is only in the logarithmic divergences of diagrams, which are dominated by gluon momenta satisfying the limit $\Lambda<<q<<\mu$, due to the form of Landau damping. (i.e., $\Lambda \sim \Delta$, while the dominant momentum transfer is $q \sim \Delta^{1 / 3} m_{D}^{2 / 3}$.) In this limit the results for $\mathrm{F}$ an $\mathrm{G}$ given in (9) are accurate.

Finally, we mention the issue of screening due to the diquark condensate itself, which is necessary for a self-consistent description of the region near the FS [16, 17]. In a conventional superconductor the relative size of the magnetic penetration depth $\lambda$ and the correlation length $\xi \sim \Delta^{-1}$ determine whether one is in the type I $(\xi>>\lambda)$ or type II $(\lambda<<\xi)$ regime. In a type II superconductor the magnetic screening length can be computed using the London formula, and is proportional to the total density of superconducting particles. In our case this would lead to a rather large effective screening mass $\lambda^{-1} \sim g^{2} \mu^{2}$ relative to the gap size. However, in high density quark matter we are actually in the type I, or Pippard, regime. In this regime the effective screening mass is much smaller, and scales with the gap $\Delta$. A direct calculation of the gluon vacuum polarization using the quark propagator in the presence of a gap (see (31) below) shows that the London-type screening applies only to long wavelength gluons with momentum less than $\sim \Delta$. Harder gluons experience a much smaller screening of the Pippard type. It is easy to see that these effects are too small to affect our RG calculation; their contributions are dominated by Landau damping effects. 


\section{RG Equations}

To obtain the RG equations we need to evaluate the scale-dependent quantum corrections to the form-factor $G(k-q)$. Let us consider the effect of integrating out quark states in the momentum shell $\Lambda^{\prime}<|\vec{q}|-\mu<\Lambda$, and gluon states in the momentum shell $\Lambda^{\prime}<|\vec{q}|<\Lambda$. The tree level contribution comes directly from one-gluon exchange, figure 1, while the one loop contribution comes from the box diagram, figure 3. It is important to note that only one topology of the box diagram contributes. The diagram with "crossed" gluon lines does not have the same kinematic structure as an iterated Cooper pairing interaction, and is subleading. Actually, in our effective theory the box diagram contains several different contributions. The most important contains two local four fermion interactions, and is actually a "bubble" diagram, with running form factor coefficients. These coefficients contain the effects of previously integrated shells of quarks and gluons. The other contributions involve at least one exchange of a soft gluon within the momentum shell, and are suppressed in the thin-shell limit. Thus, as we discuss below, the result of the one loop part of our calculation is essentially the same as iterating bubbles with form factor vertices.

Solving the RG equations is equivalent to summing up an infinite series of ladder graphs corresponding to nearly colinear scattering mediated by gluon exchange. This corresponds to the "rainbow" approximation in which the gap equation is solved in section 5.

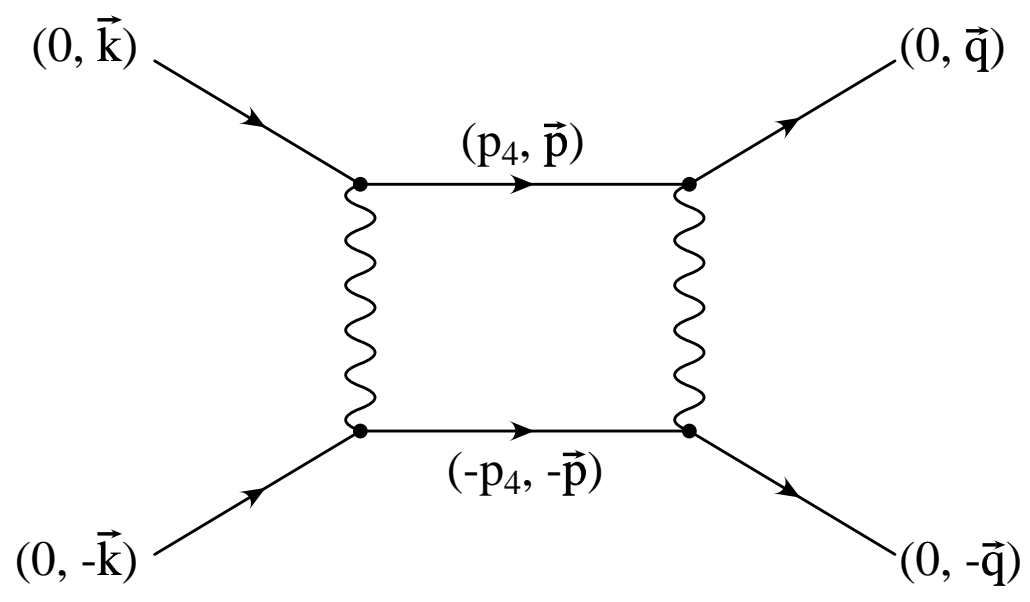

Figure 3: Box diagram.

Let us elaborate on why the tree and box graphs are sufficient to compute the leading order solution to the RG equations. Consider integrating out all modes between an upper cutoff $\sim \mu$ and a lower cutoff given by $\Delta$. For simplicity, we can consider doing this in a single step, rather than shell by shell. A systematic expansion for the result is possible in powers of the gauge coupling $g$ and powers of $t \sim \ln \Lambda$. Since the RG evolution terminates 
at a Landau pole of order $\sim \exp (-c / g), t \sim 1 / g$ in our counting scheme. As we shall see below, (figure (1) is of order $g^{2} t$, and so contributes a term of order $g^{2}$ to the RG equation. The box diagram (figure [3) is of order $g^{4} t^{3}$ and so contributes terms of order $g^{4} t^{2} \sim g^{2}$. Higher order loop corrections to either of these graphs (e.g. from vertex or wavefunction renormalization) are suppressed by at least $g^{2} t \sim g$. Of course, as discussed in the previous section, the screened gluon propagators must be used in our computations, since some of the logarithms which arise are due to small angle scattering. Additional radiative corrections beyond vacuum polarization effects on the gluon propagators are suppressed by at least a power of $g^{2} t$.

Because the rotational $\mathrm{SO}(4)$ symmetry is broken to $\mathrm{SO}(3)$, the coupling in (3) will split into its "temporal" $G_{4}$ and "spatial" $G_{i}$ parts, which we refer to as A and B components, according to the notation previously used in [5]

$$
\begin{aligned}
& A(k-q) \bar{\psi}_{+}\left(k_{0}, k\right) \gamma_{4} P_{L} \psi_{+}\left(q_{0}, q\right) \bar{\psi}_{+}\left(k_{0},-k\right) \gamma^{4} P_{L} \psi_{+}\left(q_{0},-q\right)+ \\
& B(k-q) \bar{\psi}_{+}\left(k_{0}, k\right) \gamma_{i} P_{L} \psi_{+}\left(q_{0}, q\right) \bar{\psi}_{+}\left(k_{0},-k\right) \gamma^{i} P_{L} \psi_{+}\left(q_{0},-q\right)
\end{aligned}
$$

Due to the form of the screening effects described in the previous section it is easy to see that the RG equations for the $A$-type couplings will not contain terms of order $g^{2}$. The Debye mass $m_{D}$ in the longitudinal part of the gluon propagator (17) removes any IR logarithm from the one gluon exchange diagram.

In contrast, the $B$ coupling may recieve such corrections. The leading contribution will come from the region of shell integration where one may neglect the Landau damping term $G(Q)$ in gluon propagator (7). In this region the scattering angle satisfies the following condition: $1-x^{2 / 3}<z=\cos \theta<1-x^{2 / 3}$, with $x=\frac{\pi}{2^{5 / 2}} \frac{m_{D}^{2} \Lambda}{\mu^{3}}$. The angular momentum l-component $B_{l}$ receives the contribution

$$
B_{l}(\Lambda)=-\frac{1}{3} \frac{2}{3} \frac{g^{2}}{4 \mu^{2}} \int_{-1}^{1-x^{2 / 3}} \frac{d z}{1-z} P_{l}(z)
$$

(the factor of $1 / 3$ corresponds to the attractive $\overline{3}$ channel, while the factor of $2 / 3$ arises from the transversality of the magnetic gluon propagator). The correction is of the form $\delta B_{l} \sim \frac{g^{2}}{\mu^{2}} \delta t$, proportional to the RG scaling parameter $\delta t=\ln \frac{\Lambda}{\Lambda^{\prime}}$. Note that the leading term in $\delta B_{l}$ does not depend on the details of the angular cut-offs - that ambiguity is cancelled in the definition of $t$.

The box diagram of figure 3 has the following integral representation:

$$
I(t)=-g^{4} \int \frac{d^{4} p}{(2 \pi)^{4}} K_{\mu \rho, \nu \kappa} \gamma^{\mu}\left[\left(i \mu+p_{4}\right) \gamma^{4}+\vec{p} \cdot \vec{\gamma}\right] \gamma^{\nu} \otimes \gamma^{\rho}\left[\left(i \mu-p_{4}\right) \gamma^{4}-\vec{p} \cdot \vec{\gamma}\right] \gamma^{\kappa}
$$

\footnotetext{
${ }^{2}$ For notational simplicity, we suppress the chiral projectors $P_{L}$ in our expressions, although they appear in our calculations.
} 
where $K_{\mu \rho, \nu \kappa}$ incorporates both fermion and propagators (7):

$$
K_{\mu \rho, \nu \kappa}=\frac{D_{\mu \rho}\left(p_{4}, \vec{k}-\vec{p}\right)}{\left(i \mu+p_{4}\right)^{2}+\vec{p}^{2}} \frac{D_{\nu \kappa}\left(p_{4}, \vec{p}-\vec{q}\right)}{\left(i \mu-p_{4}\right)^{2}+\vec{p}^{2}} .
$$

In order to evaluate the contribution to $\mathrm{I}(\mathrm{t})$ one should apply the familiar decomposition of momenta near FS: $\vec{p}=\vec{\mu}_{p}+\vec{p}_{n}, \vec{k}=\vec{\mu}_{k}, \vec{q}=\vec{\mu}_{q}$ with normal component being integrated in the limits $\Lambda^{\prime 2}<p_{n}^{2}<\Lambda^{2}$ [5]. Note that due to screening effects the dominant regions of $p_{n}$ and $p_{4}$ are around the origin, close to the FS.

Consider, for example, the part of $I(t)$ with both gluon lines being transverse. All other cases are done analogously. Capturing only the leading $O\left(\ln ^{3} \Lambda\right)$ behavior one gets

$$
I(t)_{T} \simeq \frac{g^{4}}{16 \mu^{2}} \iint \frac{d p_{4} d p_{n}}{(2 \pi)^{4}} \int d \Omega_{p} \frac{\gamma_{i} \gamma^{4} \gamma_{j} \otimes \gamma^{i} \gamma^{4} \gamma^{j}+\gamma_{i}(\vec{p} / \mu \cdot \vec{\gamma}) \gamma_{j} \otimes \gamma^{i}(\vec{p} / \mu \cdot \vec{\gamma}) \gamma^{j}}{\left(p_{4}^{2}+p_{n}^{2}\right)\left(1-z_{1}\right)\left(1-z_{2}\right)}
$$

where $z_{1}$ is the cosine of the angle between $\overrightarrow{\mu_{k}}$ and $\overrightarrow{\mu_{p}}$, and $z_{2}$ is the cosine of the angle between $\overrightarrow{\mu_{q}}$ and $\overrightarrow{\mu_{p}}$. Then, use the definition (15) of $B_{l}$ in the expansion of transverse gluon propagators

$$
\frac{1}{1-z}=-\frac{12 \mu^{2}}{g^{2}} \sum_{l}(2 l+1) B_{l} P_{l}(z)
$$

on the interval $]^{3} 1<z<1-x^{2 / 3}$. Applying orthogonality conditions :

$$
\begin{aligned}
\int d \Omega P_{l}\left(z_{1}\right) P_{l^{\prime}}\left(z_{2}\right) & =\delta_{l l^{\prime}} \frac{4 \pi}{2 l+1} P_{l}(z) \\
\int d \Omega \hat{p_{i}} \hat{p}_{j} P_{l}\left(z_{1}\right) P_{l^{\prime}}\left(z_{2}\right) & =\delta_{l l^{\prime}} \frac{\delta_{i j}}{3} \frac{4 \pi}{2 l+1} P_{l}(z),
\end{aligned}
$$

one obtains the final answer for the $I(t)_{T}$ in the leading divergence approximation

$$
I(t)_{T} \simeq-\frac{\mu^{2}}{4 \pi^{2}}\left(5 \gamma_{4} \otimes \gamma^{4}+\frac{13}{3} \gamma_{i} \otimes \gamma^{i}\right) B_{l}^{2}(\Lambda) t
$$

The final answer for the (Minkowskian) RGE equations is

$$
\begin{aligned}
\frac{d A_{l}}{d t} & =-\frac{N}{2}\left(A_{l}^{2}-2 A_{l} B_{l}+5 B_{l}^{2}\right) \\
\frac{d B_{l}}{d t} & =\frac{N}{6}\left(A_{l}^{2}-10 A_{l} B_{l}+13 B_{l}^{2}\right)+\frac{g^{2}}{27 \mu^{2}},
\end{aligned}
$$

where $N=\mu^{2} / 2 \pi^{2}$. These $\mathrm{RG}$ equations are the same as obtained previously for the case of local interactions [5], except for the constant term in the $B_{l}$ sector. A simple way to understand this is as follows: by expanding the four quark form factor in terms of orthogonal

\footnotetext{
3 The longitudinal gluon propagators may be expanded in Legendre polynomials $P_{l}(z)$ on the interval $-1<z<1$.
}

${ }^{4}$ The averaging over coordinate systems is assumed in the second orthogonality condition of (20). 
local operators $G_{l}$, we reduce the problem to one in which the gluon exchanges are replaced by local interactions. Hence the analysis of [5] should apply and the same RG equations obtained for each value of 1 . This is true as well for LR helicity scattering, which we do not consider explicitly here. Note that though the $A_{l}$ couplings originate in the Debye screened sector, it would be a mistake to discard them in the RGE equations due to the fact that there is mixing with the $B_{l}$, which diverge near the FS. Diagonalization of (22) gives

$$
\begin{aligned}
\frac{d}{d t}\left(A_{l}-3 B_{l}\right) & =-N\left(A_{l}-3 B_{l}\right)^{2}-\frac{g^{2}}{9 \mu^{2}} \\
\frac{d}{d t}\left(A_{l}+B_{l}\right) & =-\frac{N}{3}\left(A_{l}+B_{l}\right)^{2}+\frac{g^{2}}{27 \mu^{2}} .
\end{aligned}
$$

It is straightforward to solve these equations. For convenience, we define the spin 0 combination $S_{l} \equiv A_{l}-3 B_{l}$ and the spin 1 combination $T_{l} \equiv A_{l}+B_{l}$. We find that $S_{l}$ reaches a Landau pole at the scale

$$
\Delta \sim \mu g^{-5} \exp \left(-\frac{3 \pi^{2}}{\sqrt{2} g}\right),
$$

which is agrees with the result (迆, despite differences between the RG equations of [7] and ours (see below). $T_{l}$, due to the opposite sign of the $g^{2}$ term, does not diverge and reaches the asymptotic value of $T_{l}(t \rightarrow \infty)=\frac{\sqrt{2} \pi g}{3 \mu^{2}}$.

In order to compare our results with those of [7], it is necessary to convert four quark operators into scattering amplitudes. There are additional angular dependences introduced by the spin angular momentum of the quarks. Let us classify amplitudes by their total angular momentum, which is the sum of the spin and orbital components. Thus, for example, the $\mathrm{j}=0$ channel receives contributions from both the $\mathrm{l}=0, \mathrm{~s}=0$ and $\mathrm{l}=1, \mathrm{~s}=1$ operators. First we note that the spinor part of operators of type A introduce an additional factor of $2 \cos ^{2} \frac{\theta}{2}$ to the scattering amplitude, while the type B operators introduce a factor of $2 \cos ^{2} \frac{\theta}{2}+$ $4 \sin ^{2} \frac{\theta}{2}$. This leads to the following expression for the amplitude

$$
f(\theta)=\sum_{l}(2 l+1)\left[S_{l}+T_{l} \cos \theta\right] P_{l}(\cos \theta)
$$

The components of total angular momentum $\mathrm{j}$ are given by

$$
f_{j}=\left[S_{j}+\frac{j}{2 j+1} T_{j-1}+\frac{j+1}{2 j+1} T_{j+1}\right],
$$

where we have used the identity

$$
(2 l+1) z P_{l}(z)=(l+1) P_{l+1}(z)+l P_{l-1}(z) .
$$

The RG equations for $f_{j}$ can be deduced easily from (26) and (23). For the lowest component, we have

$$
\frac{d}{d t} f_{0}=-\frac{2 g^{2}}{27 \mu^{2}}-N S_{0}^{2}-\frac{N}{3} T_{1}^{2} .
$$


The equations for higher components are of the form

$$
\frac{d}{d t} f_{j}=-\frac{2 g^{2}}{27 \mu^{2}}-N S_{j}^{2}-\frac{N}{3}\left(\frac{j}{2 j+1} T_{j-1}^{2}+\frac{j+1}{2 j+1} T_{j+1}^{2}\right) .
$$

Several remarks are in order.

- These results are similar to, but different from, those of [7]. In particular, we do not find that the RG equations take the simple form [7]

$$
\frac{d}{d t} f_{j}=-\frac{g^{2}}{9 \mu^{2}}-N f_{j}^{2}
$$

The rhs of our equation (29) cannot be organized in terms of any simple amplitude $f_{j}^{2}$. We believe that the treatment of quantum corrections in section 2 of that paper is too crude. In particular, iterating the amplitude $f(p, k)$ in order to obtain the $f_{j}^{2}$ terms in the $\mathrm{RG}$ equation neglects some important spinor structure of the vertex which our calculation takes into account.

- Fermi statistics constrain the operators as follows. We restrict ourselves to the color $\overline{3}$ and isospin singlet channels, which are both antisymmetric. The remaining part of the wavefunction must be antisymmetric. In the antisymmetric $s=0$ channel, we must therefore have $l=0,2,4, \ldots$, while in the symmetric $s=1$ channel we must have $l=1,3,5, \ldots$. Thus the operators $S_{l}$ vanish for odd 1 and $T_{l}$ vanish for even l. From (26) we see that $f_{j}$ vanishes for all odd values of $\mathrm{j}$. Note that this analysis is modified in the LR channels, which we do not consider here.

- It is not necessary to solve the $f_{j} \mathrm{RG}$ equations, as their behavior can be deduced from that of $S_{l}$ and $T_{l}$. Since $T_{l}$ never diverges, near the FS $f_{j} \approx S_{j}$ and diverges at the scale

(24). Note that the $\frac{N}{3} T_{j}^{2}$ term cannot be neglected in (29); its asymptotic value is $\frac{g^{2}}{27 \mu^{2}}$.

- Because the Landau poles in all the $\mathrm{j}$ channels are the same, we naively expect to find condensates with non-zero angular momentum, leading to the breaking of rotational symmetry [7]. However, we will see in the next section by studying the gap equation that this is not the case.

\section{Rotational Symmetry Breaking and Gap Equation}

In this section we explore the issue of condensates with non-zero angular momentum using the gap equation. Our RG results suggest that the gap function could violate rotational invariance. Some recent papers have studied the s-wave condensate using the non-local gap equation, including the effects of magnetic gluons [16, 17, 18]. We will generalize their 
approach to consider gap functions $\Delta\left(q_{0}, \vec{q}\right)$ which are functions of orientation. Remarkably, it is possible to show that in the leading order approximation only an s-wave condensate is formed.

Let us introduce a two component field $\Psi=\left(\psi, \bar{\psi}^{T}\right)$. The inverse quark propagator takes the form

$$
S^{-1}(q)=\left(\begin{array}{cc}
\not q+\not h-m & \bar{\Delta} \\
\Delta & (\not q+\not h+m)^{T}
\end{array}\right),
$$

where $\bar{\Delta}=\gamma_{0} \Delta^{\dagger} \gamma_{0}$. The gap is a matrix in color, isospin, and Dirac space, and in our analysis we allow it to depend on orientation. As discussed, the RG analysis shows that the condensate will form in the LL (RR) channels. Given this, the form of the gap matrix is [1, 8]

$$
\Delta_{i j}^{a b}(q)=\left(\lambda_{2}\right)^{a b}\left(\tau_{2}\right)_{i j} C \gamma_{5}\left(\Delta_{+}\left(q_{0}, \vec{q}\right) \frac{1}{2}(1+\vec{\alpha} \cdot \hat{q})+\Delta_{-}\left(q_{0}, \vec{q}\right) \frac{1}{2}(1-\vec{\alpha} \cdot \hat{q})\right) .
$$

In our present weak coupling discussion, we are only interested in $\Delta_{+} \cdot \Delta_{-}$corresponds to a condensate of anti-quarks, and does not influence $\Delta_{+}$. Henceforth we shall only refer to $\Delta \equiv \Delta_{+}$. Making the usual FS approximations, the gap equation has the form

$$
\Delta\left(q_{0}, q, \hat{q}\right)=-i g^{2} \int \frac{d^{4} k}{(2 \pi)^{4}} \frac{D\left(q_{0}-k_{0}, \vec{q}-\vec{k}\right) \Delta\left(k_{0}, k, \hat{k}\right)}{\epsilon(k)^{2}+k_{0}^{2}+\left|\Delta\left(k_{0}, k, \hat{k}\right)\right|^{2}}
$$

where the interaction kernel $D\left(q_{0}-k_{0}, \vec{q}-\vec{k}\right)$ is essentially the gluon propagator up to some additional factors arising from the particle projector and gamma matrices. Here $k=|\vec{k}|$, $q=|\vec{q}|$ and $\epsilon(k)=k-\mu$; we assume $q_{0}^{2} \sim q_{0}^{2}<<\mu^{2}$ and $k \sim q \simeq \mu$. For the next step in our analysis it is useful to separate the interaction kernel into angular momentum channels,

$$
D\left(q_{0}-k_{0}, \vec{q}-\vec{k}\right)=\sum_{l}(2 l+1) P_{l}\left(\cos \theta_{k q}\right) D^{l}\left(q_{0}-k_{0},|\vec{q}-\vec{k}|\right) .
$$

The $D^{l}$ coefficients are obtained by integrating the kernel against Legendre polynomials $P_{l}(\cos \theta)$. Due to the divergence at small angle, all of the $D^{l}$ have the same value at leading logarithmic order.

The gap equation can now be rewritten as

$$
\Delta\left(q_{0}, q, \hat{q}\right)=-4 \pi i g^{2} \sum_{l m} Y_{m}^{l}\left(\theta_{q}, \phi_{q}\right) \int \frac{d^{4} k}{(2 \pi)^{4}} Y_{m}^{l *}\left(\theta_{k}, \phi_{k}\right) \frac{D^{l}\left(q_{0}-k_{0},|\vec{q}-\vec{k}|\right) \Delta\left(k_{0}, k, \hat{k}\right)}{\epsilon^{2}(k)+k_{0}^{2}+\left|\Delta\left(k_{0}, k, \hat{k}\right)\right|^{2}} .
$$

For $D^{l}=D\left(q_{0}-k_{0},|\vec{q}-\vec{k}|\right)$ independent of 1 , the sum over spherical harmonics reduces to a delta function: $\delta\left(\phi_{q}-\phi_{k}\right) \delta\left(\cos \theta_{q}-\cos \theta_{k}\right)$. The angular integral on the rhs of the equation can be trivially evaluated, yielding

$$
\Delta\left(q_{0}, q, \hat{q}\right)=-i g^{2} \int \frac{d k_{4} d k k^{2}}{(2 \pi)^{3}} \frac{D\left(q_{0}-k_{0},|\vec{q}-\vec{k}|\right) \Delta\left(k_{0}, k, \hat{q}\right)}{\epsilon^{2}(k)+k_{0}^{2}+\left|\Delta\left(k_{0}, k, \hat{q}\right)\right|^{2}} .
$$


This equation can be regarded as a set of identical equations, one for each orientation $\hat{q}$. Since each equation is identical, the solution must be independent of orientation.

This result could have been guessed directly from the fact that colinear scattering dominates the magnetic effects. In that approximation the kernel $D$ is proportional to a delta function $\delta(\hat{k}-\hat{q})$, and it is clear that (33) only has an s-wave solution.

Our analysis thus far has been within the leading logarithmic approximation. We can relax this condition by considering a gap function

$$
\Delta(q)=\Delta^{0}+\Delta^{1}\left(q_{0}, \vec{q}\right)
$$

where $\Delta^{0}$ is the constant (in orientation) solution obtained from (33), and $\Delta^{1}$ is a small perturbation which can depend on orientation. We will show that $\Delta^{1}$ is at most of order $\exp \left(-c / g^{2}\right)$ and hence negligible relative to the $\Delta^{0}$. Substituting (37) into (33), we obtain two gap equations. The leading order equation is the just the usual one for an s-wave condensate, and determines $\Delta^{0}$. Note that we retain the complete interaction kernel $D\left(q_{0}-k_{0}, \vec{q}-\vec{k}\right)$ here, without making the leading log approximation. The second equation contains terms of $\mathcal{O}\left(\Delta^{1}\right)$ :

$$
\Delta^{1}\left(q_{0}, \vec{q}\right) \simeq g^{2} \mu^{2} \int \frac{d k_{0} d \Omega_{k q}}{(2 \pi)^{3}} \frac{D\left(q_{0}-k_{0}, \theta_{k q}\right)}{2 \sqrt{k_{0}^{2}+\left|\Delta^{0}\right|^{2}}}\left[1-\frac{\left|\Delta^{0}\right|^{2}}{k_{0}^{2}+\left|\Delta^{0}\right|^{2}}\right] \Delta^{1}\left(k_{0}, \vec{k}\right)
$$

This equation was obtained after first performing the integral over $\mathrm{k}$, in the approximation that $\Delta$ and $D$ are slowly varying for $k \simeq \mu$. The term in brackets in (38) suppresses the logarithmic divergence in the integral over $k_{0}$ near the FS, although there is still a potential divergence from the small angle behavior of $D\left(q_{0}-k_{0}, \theta_{k q}\right)$. Hence the solution $\Delta^{1}$ is at most of order $\exp \left(-1 / g^{2}\right)$, and is negligible relative to $\Delta^{0}$ in the weak coupling limit.

The discussion in terms of $\Delta^{0}$ and $\Delta^{1}$ is quite general. We can also apply it to lower densities, where the magnetic interaction is presumably screened by non-perturbative effects, and quark interactions are effectively local. In this case the largest condensate can be shown to be rotationally invariant [1, 5]. Because $D$ in (38) is non-singular, a non-zero solution $\Delta^{1}$ only exists above some critical coupling (if at all). This is unlike the usual Cooper pairing instability in which an arbitrarily weak interaction can lead to a condensate. It seems that a large s-wave component tends to inhibit condensates of higher angular momentum.

Having made some general observations about rotational invariance, we now concentrate on solving the gap equation explicitly, in order to check our result for the Landau pole (24). The authors of 116, 17, 18] do not consider higher orbital angular momentum components $\Delta_{l>0}$ in their analyses, but it is straightforward to do so. 
The explicit gap equation for $\Delta_{1} \equiv \Delta$, neglecting the suppressed antiparticle contribution, is [17, 18]

$$
\Delta\left(q_{0}, \vec{q}\right)=-\frac{2 i g^{2}}{3} \int \frac{d^{4} k}{(2 \pi)^{4}} \frac{\Delta\left(k_{0}, \vec{k}\right)}{\epsilon^{2}(k)+k_{0}^{2}+\left|\Delta\left(k_{0}, \vec{k}\right)\right|^{2}} \frac{\frac{3}{2}-\frac{1}{2} \hat{k} \cdot \hat{q}}{(k-q)^{2}+G} .
$$

Here we have written explicitly the factors resulting from the particle projector and gamma matrices. $\mathrm{G}$ is the Landau damping term which appears in the magnetic gluon propagator; we neglect the effect of the electric gluon.

In order to consider higher angular modes of $\Delta$ we make the expansion:

$$
\Delta\left(q_{0}, \vec{q}\right)=\sum_{l}(2 l+1) P_{l}(\hat{q}) \Delta_{l}
$$

as well as a similar expansion of the gluon propagator with coefficients

$$
D_{l}=\frac{1}{2 \mu^{2}} \int_{-1}^{1} d z \frac{P_{l}(z)}{1-z+G /\left(2 \mu^{2}\right)}
$$

which are all of the same size at leading order. Using the identity

$$
P_{l}\left(\cos \theta_{k q}\right)=\frac{2 \pi}{2 l+1} \sum_{m=-l}^{m=l} Y_{l}^{m}\left(\theta_{k}, \phi_{k}\right)^{*} Y_{l}^{m}\left(\theta_{q}, \phi_{q}\right)
$$

we obtain the following coupled gap equations

$$
\begin{aligned}
\Delta_{l} & =-\frac{2 i g^{2}}{3 \pi} \int \frac{d k_{0} d k}{(2 \pi)^{2}} \frac{\mu^{2}}{\epsilon^{2}(k)+k_{0}^{2}+\left|\Delta\left(k_{0}, \vec{k}\right)\right|^{2}} \\
& \times \quad\left[\frac{3}{2} D_{l} \Delta_{l}-\frac{1}{2(2 l+1)}\left((l+1) D_{l+1} \Delta_{l}+l D_{l-1} \Delta_{l}\right)\right] .
\end{aligned}
$$

In obtaining this equation we have neglected the angular dependence of $\left|\Delta\left(k_{0}, \vec{k}\right)\right|^{2}$ which occurs in the denominator. This is justified if the gap turns out to be rotationally invariant, as expected from our previous arguments. It is easy to see that a self-consistent solution exists with all $l>0$ gaps zero, and the solution for $\Delta=\Delta_{0}$ given by (24).

\section{Discussion}

In this paper we investigated the renormalization group behavior of QCD at high density, concentrating on the effects of long range magnetic interactions. Our approach was somewhat different from that of [7] in that we focused on individual local operators rather than scattering amplitudes. The resulting RG equations are different, although the location of 
the Landau pole is still given by (11). The disagreement results from two causes: (I) the transverse form of the propagator does not appear to have been used in [7], leading to a different coefficient in the constant $g^{2}$ term of the RGE and (II) the treatment of spinor properties of the quantum corrections is different in the two calculations. We believe that the renormalization group applied directly to amplitudes does not properly compute the loop corrections. Some additional issues we attempted to clarify include the validity of the use of Landau damping in resummed gluon propagators, the gauge invariance of the computation and the size of subleading corrections. Our RG equations (23) are gauge invariant, and represent the leading order result in a self-consistent expansion. Corrections to the coefficients in (23) are of order $\mathcal{O}(g)$ in the weak coupling limit.

We also used the gap equation to investigate whether rotational symmetry is broken at asymptotically high densities. The gap equation analysis shows that scattering which is predominantly colinear leads to a rotationally invariant condensate. We found, in disagreement with a naive interpretation of the RG results, that any condensates of higher angular momentum are exponentially smaller than the s-wave condensate. We also checked that the solution of the gap equation agrees with the value of our Landau pole.

The authors would like to thank Nick Evans, Deog-Ki Hong, James Hormuzdiar, Rob Pisarski and Dirk Rischke for useful discussions and comments. This work was supported in part under DOE contracts DE-FG02-91ER40676 and DE-FG06-85ER40224.

\section{References}

[1] D. Bailin and A. Love, Nucl. Phys. B190 (1981) 175; Nucl. Phys. B190 (1981) 751; Nucl. Phys. B205 (1982) 119; Phys. Rep. 107 (1984) 325).

[2] M. Alford, K. Rajagopal and F. Wilczek, Phys.Lett. B422, 247 (1998).

[3] R. Rapp, T. Schafer, E. V. Shuryak and M. Velkovsky, Phys.Rev.Lett.81, 53 (1998).

[4] R. Rapp, T. Schafer, E. V. Shuryak and M. Velkovsky, hep-ph/[A9904353.

[5] N. Evans, S. D. H. Hsu and M. Schwetz, Nucl.Phys.B551, 275 (1999); Phys.Lett.B449, 281 (1999).

[6] T. Schafer and F. Wilczek, Phys.Lett.B450:325-331,1999; hep-ph/9810509.

[7] D. T. Son, Phys.Rev.D54 094019 (1999). 
[8] R. D. Pisarski and D. H. Rischke, nucl-th/9811104.

[9] D. K. Hong, hep-ph/9812510.

[10] D. K. Hong, hep-ph/9905523.

[11] D. K. Hong, M. Rho and I. Zahed, hep-ph/9906551.

[12] M. Alford, K. Rajagopal and F. Wilczek, Nucl.Phys.B537, 443 (1999).

[13] J. Berges and K. Rajagopal, Nucl.Phys.B538, 215 (1999).

[14] S. Hands, J.B. Kogut, M.-P. Lombardo and S.E. Morrison, hep-lat/9902034; S. Hands and S.E. Morrison hep-lat/9902012, hep-lat/990521. J.B. Kogut, M.A. Stephanov and D. Toublan, hep-ph/9906346.

[15] T. Schafer and F. Wilczek, PRL 82 (1999) 3956; hep-ph/9903503.

[16] D.K. Hong, V.A. Miransky, I.A. Shovkovy and L.C.R. Wijewardhana, hep-ph/9906478.

[17] T. Schafer and F. Wilczek, hep-ph/9906512.

[18] R. D. Pisarski and D. H. Rischke, nucl-th/9907041.

[19] R. Shankar, Physica A177, 530 (1991); Rev. Mod Phys. 66, 129 (1993).

[20] M. Le Bellac, Thermal Field Theory, Cambridge University Press, Cambridge, 1996. 